\title{
Review Article \\ Uveitis and Gender: The Course of Uveitis in Pregnancy
}

\author{
Nathalie P. Y. Chiam and Lyndell L. P. Lim \\ Centre for Eye Research Australia, University of Melbourne, Royal Victorian Eye and Ear Hospital, 32 Gisborne Street, \\ East Melbourne, VIC 3002, Australia
}

Correspondence should be addressed to Lyndell L. P. Lim; limllp@unimelb.edu.au

Received 25 September 2013; Accepted 9 December 2013; Published 9 January 2014

Academic Editor: H. Nida Sen

Copyright (C) 2014 N. P. Y. Chiam and L. L. P. Lim. This is an open access article distributed under the Creative Commons Attribution License, which permits unrestricted use, distribution, and reproduction in any medium, provided the original work is properly cited.

\begin{abstract}
The hormonal and immunological changes in pregnancy have a key role in maintaining maternal tolerance of the semiallogeneic foetus. These pregnancy-associated changes may also influence the course of maternal autoimmune diseases. Noninfectious uveitis tends to improve during pregnancy. Specifically, uveitis activity tends to ameliorate from the second trimester onwards, with the third trimester being associated with the lowest disease activity. The mechanism behind this phenomenon is likely to be multifactorial and complex. Possible mechanisms include Th1/Th2 immunomodulation, regulatory T-cell phenotype plasticity, and immunosuppressive cytokines. This clearly has management implications for patients with chronic sight threatening disease requiring systemic treatment, as most medications are not recommended during pregnancy due to lack of safety data or proven teratogenicity. Given that uveitis activity is expected to decrease in pregnancy, systemic immunosuppressants could be tapered during pregnancy in these patients, with flare-ups being managed with local corticosteroids till delivery. In the postpartum period, as uveitis activity is expected to rebound, patients should be reviewed closely and systemic medications recommenced, depending on uveitis activity and the patient's breastfeeding status. This review highlights the current understanding of the course of uveitis in pregnancy and its management to help guide clinicians in managing their uveitis patients during this special time in life.
\end{abstract}

\section{Introduction}

Pregnancy is associated with various hormonal and immunological changes that facilitate the survival of the semiallogeneic foetus. These physiological changes influence the course of various maternal autoimmune diseases $[1,2]$. The effect of pregnancy on noninfectious uveitis has not been as extensively studied; however, to date it has been well described by a few authors. It is essential to understand the course of uveitis in pregnancy as uveitis has a peak incidence in young adults and it is not uncommon for female patients with known uveitis to become pregnant. This review will examine the literature on the course of uveitis in pregnancy and its management. This summary would hopefully help guide clinicians in the management of uveitis during pregnancy and the postpartum period.

\section{Theories on How Pregnancy Influences Uveitis}

During pregnancy, the tolerance of the semiallogeneic foetus is made possible by the various hormonal and immunological changes in pregnancy. These physiological changes also have a role in influencing the course of maternal autoimmune diseases $[1,2]$.

The increased levels of oestrogen and progesterone during pregnancy result in the suppression of Th1 associated immunity but the upregulation of Th2 associated immune responses [3-5]. As such, pregnancy often ameliorates Th1 associated autoimmune diseases, like rheumatoid arthritis, but exacerbates Th2 associated autoimmune conditions, like systemic lupus erythematosus [2-9]. The association between uveitis amelioration and Th1 suppression/Th2 upregulation 
has been demonstrated by serum studies in Chan et al.s [10] prospective case study on four pregnant uveitis patients. Agarwal et al. [11] have also reported similar findings for experimental autoimmune uveitis (EAU) in mice. When EAU susceptible mice $(\mathrm{C} 57 \mathrm{BL} / 6)$ were immunised with interphotoreceptor retinoid binding protein, the incidence and severity of EAU were lower in the pregnant mice, as compared to nonpregnant controls. The pregnant mice were also found to have reduced levels of interferon gamma, IL 12 P40 but unchanged levels of TNF alpha, IL4, IL5, and IL10, which suggested a Th2 bias in their immune system [11]. This Th2 bias in pregnancy probably augments the Th1 predominant response in noninfectious uveitis, resulting in disease amelioration [12]. Although still uncertain, the recently discovered subset of $\mathrm{T}$ helper cells, Th17, may also play a role in altered autoimmune activity in pregnancy [1317]. Th17 cells are proinflammatory and associated with the pathogenesis of autoimmune diseases like systemic lupus erythematosus [18],Vogt-Koyanagi-Harada (VKH) disease [19], irritable bowel disease [20], rheumatoid arthritis [21], and multiple sclerosis [22]. During pregnancy, Th17 cells are elevated in preeclampsia $[9,23]$. The hormonal and associated cytokine changes in pregnancy influence autoimmune disease activity and may inspire future therapeutic options. Interestingly, studies have shown that oral oestradiol may decrease disease activity in multiple sclerosis [24, 25]; however, its implications in uveitis management are uncertain.

Several other pregnancy-associated changes may influence the course of maternal autoimmune conditions. For instance, regulatory $\mathrm{T}$ cells demonstrate phenotype plasticity and are able to switch between a tolerant or aggressive phenotype in response to circulating foetal cells or infectious agents accordingly $[17,26]$. The elevated levels of immunosuppressive cytokines and hormones, such as melanocytestimulating hormone [27, 28], early pregnancy factor [29], and alpha-fetoprotein [30,31] have also been implicated in the improvement of various autoimmune conditions during pregnancy. The mechanism for altered activity of autoimmune uveitis in pregnancy is likely to be multifactorial.

The available literature seems to suggest that uveitis activity begins to improve in mid pregnancy and reaches its lowest level in the third trimester (see below). This may be due to the various pregnancy-associated changes, such as the Th1/Th2 immune shift, becoming increasingly pronounced with the progress of pregnancy $[6,32]$. These findings are in keeping with the accepted theory that most forms of non-infectious uveitis are Th1 mediated diseases [12]. After delivery, the rate of flare-up seems to return to prepregnancy levels. This may be explained by the reversal of various pregnancy-associated changes within one to two months of delivery [33].

\section{The Effect of Pregnancy on the Course of Uveitis}

There have only been a few studies that investigated pregnancy's effect on noninfectious uveitis. Previous publications on uveitis in pregnancy include a few case reports [10, 34-36], a retrospective case series by Rabiah and Vitale [37] in 2003, and a retrospective cohort study by Kump et al. [38] in 2006. The authors of this review have also recently conducted a retrospective case series on uveitis in pregnancy [39]. As uveitis is an uncommon condition [40], studies on uveitis in pregnancy are constrained by the limited number of eligible patients and are largely restricted to retrospective studies. The general consensus is that uveitis activity improves in pregnancy, with significantly decreased disease activity from the mid pregnancy onwards. However, in the postpartum period, uveitis activity tends to relapse.

The findings from previous case reports and small case series $(n \leq 4)$ [10, 34-36] have limited generalizability due to the small numbers of patients studied. Even so, they reported that uveitis improves in pregnancy, especially in the mid and late trimesters while postpartum period was associated with activity relapse, which was reflected by other larger studies.

The retrospective case series by Rabiah and Vitale [37] was based in Saudi Arabia. It included 76 pregnancies among 50 women. Their subjects had VKH associated uveitis (46\%), Behcet's disease associated uveitis (20\%), and idiopathic uveitis (34\%), which reflected the regional epidemiology in Saudi Arabia. The study investigated the probability of at least one flare-up in the periods three months before pregnancy, during pregnancy, and up to six months postpartum. They reported that the probability of uveitis flaring-up was lower during pregnancy as compared to three months prepregnancy and six months postpartum. It should be noted that the duration of followup in prepregnancy, pregnancy, and postpartum was unequal. As such, a larger number of patients may experience a flare-up when the duration of followup was longer; thus their findings should be interpreted with this in mind.

The retrospective cohort study by Kump et al. [38] was based in the United States of America. It involved 32 pregnant self-controls and 32 nonpregnant female controls who were matched for age, ethnicity, and anatomical location of uveitis. Most subjects had idiopathic uveitis (72\%). They reported that the annual rate of flare-up was significantly lower during pregnancy (1.0 per year) as compared to nonpregnant periods (2.4 per year) and non-pregnant controls (3.1 per year), $P<0.001$. During pregnancy, rates of flare-up decreased significantly in the second and third trimester $(2.3,0.5,0.4$ per year for the first, second, and third trimesters, resp.).

Chiam et al's retrospective study was based in Australia and included 47 subjects [39]. Uveitis activity one year prepregnancy, during pregnancy and one year postpartum was evaluated. The reported flare-up rates were 1.188, 0.540, 0.972 per person year in prepregnancy, gestation, and postpartum, respectively. ( $P<0.001$ for comparison between pre-pregnancy and pregnancy; $P=0.009$ for comparison between pregnancy and postpartum). The rate of flare-up was $1.188,0.264,0.096$ per person year for the first, second, and third trimesters, respectively. Rates in the second trimester were significantly lower than rates in the first trimester, $P=$ 0.002; meanwhile rates in the third trimester did not differ significantly from the second trimester, $P=0.338$. After delivery, rates of flare-up rebounded, as flare-up rates six months postpartum were not significantly different from prepregnancy rates $(P=0.306)$. 
Interestingly, the severity of uveitis flare-ups does not seem to be influenced by the course of pregnancy. Chiam et al. reported that when uveitis severity was evaluated based on anterior chamber cell count, the severity of flare-ups was not significantly different between pregnancy and nonpregnant periods [39]. In Rabiah and Vitale's study [37], surrogate markers of disease severity including flare-up duration and type of therapy prescribed were also not significantly different in pregnancy and nonpregnant periods.

Other factors have also been studied with regard to their possible influence on uveitis activity during this period. These include the effect of breastfeeding, the possible relationship between multiple pregnancies in the same individual and various host factors such as type of uveitis.

Lactation has been suggested to aggravate some autoimmune diseases. After delivery, elevated prolactin levels from pregnancy will decline unless breastfeeding occurs. As prolactin is a proinflammatory hormone that promotes Th1immune responses [2], Th1-dominant immunopathologies like rheumatoid arthritis have been shown to be aggravated by lactation $[8,41-45]$. Although breastfeeding has not been found to have a significant influence on the likelihood of uveitis flare-up in the postpartum period, this is likely to be due to the small numbers of subjects available for analysis [37-39]. Similarly, although uveitis activity in pregnancy does not seem to be correlated between different pregnancies within multiparous individuals $[37,39]$, the small numbers of subjects available for analysis in these studies were again limited.

In general, the course of uveitis varies across uveitis aetiologies. However, it is interesting to note that in our study, host variables such as uveitis aetiology, anatomical location of uveitis, course of uveitis activity, medication used, and sex of child were not found to be associated with flare-up rates in pre-pregnancy, gestation, or postpartum period. In particular, it is interesting to note that uveitis activity seems to improve during pregnancy across most uveitis aetiologies. This is supported by other studies that analysed the effect of pregnancy according to uveitis diagnosis, where uveitis activity was found to improve from the second trimester onwards across the various uveitis aetiologies [37, 39]. Uveitis aetiologies analysed in these studies included HLA-B27 associated uveitis, VKH disease, Behcet's disease, and idiopathic uveitis.

Articles focusing on systemic autoimmune diseases in pregnancy have also suggested that the associated uveitis tends to improve for most of these conditions $[3,4,7,41,42$, 46-52]; however, the opposite applies to systemic lupus erythematosus, where ocular inflammation has been reported to increase in pregnancy $[7,51]$. Meanwhile reports have been contradictory for VKH associated uveitis [53-58]. Rabiah and Vitale's retrospective study reported that their VKH subjects $(n=33)$ mostly experienced an early pregnancy flare-up, with approximately half experiencing a postpartum flare-up [37]. However, this has not been a consistent pattern amongst prior studies. Two case reports have described VKH patients experiencing flare-ups in mid and late pregnancy $[55,58]$. Meanwhile, other case reports have described VKH activity in early pregnancy, with cases of VKH being first diagnosed between 10 and 16 weeks [56, 57]. There have also been case reports on $\mathrm{VKH}$ generally improving during pregnancy $[53,54]$. It is therefore difficult to ascertain the course of VKH on pregnancy as the existing literature is restricted to case reports which describe inconsistent experiences.

The use of anti-inflammatory medications has not been found to be associated with rates of flare-up during pregnancy $[37,39]$. However, this may be due to selection bias, as patients who did not receive treatment probably had relatively inactive uveitis, whereas those on medication likely had more aggressive disease that required treatment. On the other hand, the lack of association could also be due to the relatively small sample sizes (type II error) in these studies.

\section{The Management of Uveitis in Pregnancy}

The management of non-infectious uveitis in pregnancy attracts special interest as non-infectious uveitis is often managed with immunosuppressive agents that may affect fertility and the viability of pregnancies. The management of uveitis in pregnant women is therefore an area of uncertainty for clinicians due to the limited information available.

Wakefield et al. [59] recently published a review on the treatment of severe inflammatory eye disease in pregnancy and young patients of reproductive age. They advised that both male and female patients should be informed about the risks of infertility, miscarriage, and foetal abnormalities. Measures to address these adverse effects of immunosuppressants include sperm banking for male patients, oocyte cryopreservation for female patients, the use of double contraception (barrier and hormonal), and enforcing a drug washout period before conception is attempted. Female patients who become pregnant should be encouraged to inform their doctors as soon as possible so that their treatment may be modified if required for the safety of the pregnancy [59]. In general, principles in the management of uveitis in pregnancy include collaboration between the obstetrician, ophthalmologist, and the patient to evaluate the risks and benefit for the mother and child $[59,60]$.

Although many immunosuppressive agents are not recommended during pregnancy due to the lack of safety data rather than due to proven teratogenicity, some have proven adverse effects on the fetus and must be avoided. Specifically, methotrexate is contraindicated during pregnancy and lactation, as it results in both miscarriage and fetal anomalies. Similarly, cyclophosphamide and mycophenolate mofetil (MMF) should also be avoided in pregnancy. MMF has been associated with a high rate of fetal anomalies and miscarriages and has therefore resulted in the development of a risk evaluation and mitigation strategy (REMS) for this drug as mandated by the Food and Drug Administration [61]. Cyclophosphamide use poses fetal malformation risks and developmental delay and is absolutely contraindicated in early pregnancy $[62,63]$. Although azathioprine and cyclosporine can be used with caution during pregnancy [63], there is currently insufficient data regarding the use of tumour necrosis factor blockers, anakinra and rituximab in pregnancy and lactation [59, $62,63]$. Table 1 summarises the current recommendations 
TABLE 1: Immunosuppressive drugs in pregnancy and lactation (adapted from reviews on immunomodulatory agents in pregnancy) [59, 62$65]$.

\begin{tabular}{lcc}
\hline Class & Side effects on pregnancy and foetus & Recommendations \\
\hline
\end{tabular}

Corticosteroids

(i) Foetal: cleft palate/lip, foetal growth retardation, adrenal suppression, neonate cataract Prednisolone $[99,100]$

(ii) Maternal: glucose intolerance, hypertension, osteopenia

(i) Food and Drug Administration Category B drug

(ii) May be used in pregnancy and breastfeeding

(iii) Ideally use prednisolone doses of $\leq 10 \mathrm{mg}$ /day

(iv) May need stress dosing

(hydrocortisone/methylprednisolone) at labour, delivery, immediate postpartum period $[63,101]$ (v) Prednisolone level in milk is $<0.1 \%$ of the prednisolone dose ingested by the mother Minimise exposure by nursing 4 hours after dose is taken if daily dose exceeds $20 \mathrm{mg}[102,103]$

Antimetabolites

(i) Foetal: the foetal liver lacks the enzyme, inosinate pyrophosphorylase, which converts

Azathioprine 6-Mercaptopurine

Methotrexate (MTX)

Mycophenolate mofetil (MMF)

Tacrolimus

(i) Foetal: infant T-, B-, NK-cell development abnormalities [110]

Cyclosporine lymphoma seem to be affected azathioprine to active metabolites; therefore the fetus is protected from the adverse effects of azathioprine (especially early pregnancy) [104] (ii) Paternal: male fertility and pregnancy do not seem to be affected [105]

(i) Foetal: miscarriage, congenital malformations (limb defects, cranial and central nervous system abnormalities) especially in first trimester (ii) Paternal: oligospermia (may be irreversible)

(i) Foetal: congenital malformations (distinctive MMF embryopathy), abortions (especially in first trimester)

(ii) Paternal: male fertility and pregnancy do not seem to be affected (i) Food and Drug Administration Category D drug

(ii) Has been used in pregnancy for many years [64]

(iii) Ideally use doses $<2 \mathrm{mg} / \mathrm{kg} /$ day. Consider decreasing dose at 32 weeks [63]

(iv) Breastfeeding is not recommended [106]

(i) Food and Drug Administration Category X drug

(ii) Cease 3 months before conception (male and females), continue folic acid after stopping MTX and during pregnancy

(iii) Not considered safe in breastfeeding due to inadequate data

(i) Food and Drug Administration Category D drug

(ii) Avoid in pregnancy

(iii) Use of MMF in pregnancy has not been widely studied; however available reports suggest avoiding MMF if possible during pregnancy [107-109]

(iv) Cease $>6$ weeks before conception attempted [63]

(v) MMF is often switched to azathioprine during pregnancy [65]

(vi) Breastfeeding is not recommended [65]

T-cell inhibitors

(ii) Maternal: renal impairment, hypertension,

(iii) Paternal: male fertility and pregnancy do not

(i) Food and Drug Administration Category C drug

(ii) May be used during pregnancy

(iii) Dosage $2.5-5 \mathrm{mg} / \mathrm{kg} /$ day — not recommended for use in breastfeeding. However, there have been reports of use in breastfeeding without adverse effects [111]

(i) Food and Drug Administration Category C drug

(ii) Insufficient information to recommend use in pregnancy

(iii) Avoid breast feeding 
TABle 1: Continued.

\begin{tabular}{|c|c|c|}
\hline Class & Side effects on pregnancy and foetus & Recommendations \\
\hline \multicolumn{3}{|c|}{ Interferon } \\
\hline Interferon-2a & (i) Foetal: not teratogenic in animal studies & $\begin{array}{l}\text { (i) Food and Drug Administration Category C } \\
\text { drug } \\
\text { (ii) American College of Paediatricians classifies } \\
\text { interferon-2a as safe in pregnancy and } \\
\text { breastfeeding } \\
\text { (iii) However, given the limited data on human } \\
\text { studies, it should be avoided in pregnancy ideally }\end{array}$ \\
\hline \multicolumn{3}{|c|}{ Anti-TNF } \\
\hline $\begin{array}{l}\text { Infliximab } \\
\text { Adalimumab } \\
\text { Etanercept }\end{array}$ & $\begin{array}{l}\text { (i) Foetal: possible risk of VACTERL (vertebral } \\
\text { anomalies, anal atresia, cardiac defects, } \\
\text { tracheoesophageal fistula, esophageal atresia, } \\
\text { renal anomalies, limb dysplasia). Currently effects } \\
\text { are still uncertain }[62,112-115] \\
\text { (ii) TNF antagonists may affect fertility [116] }\end{array}$ & $\begin{array}{l}\text { (i) Food and Drug Administration Category B } \\
\text { drug } \\
\text { (ii) Not recommended for use in pregnancy and } \\
\text { breastfeeding, unless potential benefits outweigh } \\
\text { the potential risks [63] } \\
\text { (iii) Limited data on infliximab use in lactation, } \\
\text { therefore should avoid breastfeeding (iv) Cease } \\
\text { infliximab for } 6 \text { months before starting } \\
\text { breastfeeding }\end{array}$ \\
\hline
\end{tabular}

Anti-CD 20 B-cell inhibitor

(i) Food and Drug Administration Category C drug

Rituximab (i) Foetal: case reports of granulocytopenia and

(ii) Not recommended for use in pregnancy and lymphopenia

breastfeeding, unless potential benefits outweigh the potential risks

(iii) Cease 1 year before attempting conception

Interleukin-1 receptor antagonist

$\begin{array}{ll}\text { Anakinra } & \text { (i) Foetal: no toxicity demonstrated in animal } \\ \text { studies }\end{array}$

(i) Foetal: congenital malformation (craniofacial and distal limb defects), developmental delay [117]

Cyclophosphamide (ii) Maternal: infertility, amenorrhoea, ovarian failure

(iii) Paternal: oligospermia (may be irreversible) [118-120]
Alkylating agents

(i) Food and Drug Administration Category B drug

(ii) Only use in pregnancy and lactation if needed to suppress disease activity

(i) Food and Drug Administration Category X drug

(ii) Absolutely contraindicated in the first trimester but may be used in latter half of pregnancy [64]

(iii) Cease 3 months before attempting conception (iv) Contraindicated in breastfeeding [121]

Dihydrofolate reductase inhibitor

(i) Foetal: kernicterus, agranulocytosis, no significant increase in congenital abnormalities $[62,122-124]$

(ii) Paternal: oligospermia (reversible) (i) Food and Drug Administration Category B drug

(ii) Probably safe for use in pregnancy [124] and breastfeeding $[125,126]$

Intravenous Immunoglobulin therapy

(i) Food and Drug Administration Category C drug

(ii) Good safety profile in use during pregnancy (in studies on autoimmune conditions, other than uveitis)

regarding the use of various immunosuppressive drugs in pregnancy, as advised in previous reviews [59, 62-65].

Wakefield et al's review proposed a stepwise therapeutic regimen for the management of uveitis in pregnancy according to disease severity. In mild uveitis, treatment could consist of topical or local steroid injections, followed by oral prednisolone $(<50 \mathrm{mg} /$ day $)$, azathioprine ( $2 \mathrm{mg} / \mathrm{kg} /$ day), or cyclosporine $(2.5-5 \mathrm{mg} / \mathrm{kg} /$ day $)$. Higher doses of prednisolone $(1 \mathrm{mg} / \mathrm{kg} /$ day $)$ were recommended for more severe uveitis, with the addition of azathioprine and/or 
cyclosporine if needed. In the event where triple therapy with steroids, azathioprine, and cyclosporine was insufficient for the control of inflammation, the addition of intravenous immunoglobulin therapy or biological agents could then be considered [59]. In addition, for those patients taking chronic corticosteroids during pregnancy, Wakefield et al. recommended that the dose should be increased prior to delivery $(24,12$, and 1 hour prior to delivery) to counteract the stress of childbirth.

However, as we and others have found that uveitis is generally less active during pregnancy than during the prepregnancy and postpartum periods, given the questionable safety of several systemic agents used in the treatment of noninfectious uveitis, an alternative approach would be to taper and/or cease systemic treatments during pregnancy in favour of locally delivered treatment.

The use of locally delivered treatment (such as periocular sustained release corticosteroid injections or intravitreal steroids) in non-infectious uveitis is not new and its use has been extensively described in a large range of non-infectious ocular inflammatory conditions. Periocular corticosteroids of triamcinolone and methylprednisolone have been effective in managing vitritis, posterior segment inflammation, and moderate macular oedema [66-73]. They confer the advantages of achieving higher drug levels in the posterior segment of the eye as compared to systemic steroids and lower risks of systemic side effects [74]. However, potential complications include ptosis, orbital fat protrusion, and other steroid induced ocular complications such as cataracts and raised intraocular pressure (IOP) $[66,73,75,76]$.

Intravitreal triamcinolone acetate (IVTA) is commonly used to treat vitritis and associated cystoid macular oedema [77-82]. Specifically, IVTA has been effectively used to treat uveitis associated with Behcet's disease [83-85], VKH syndrome [86], serpinginous choroiditis [87], and sympathetic ophthalmia [88-91]. These studies have shown that IVTA may be used alone or as an adjuvant to reduce the dose of systemic immunosuppression required. As compared to other forms of steroids, IVTA has been shown to be more effective than orbital floor and sub-Tenon triamcinolone [92, 93] and comparably as effective as oral steroids in managing posterior uveitis. However, side effects associated with IVTA include relatively high risks of steroid induced cataracts (15$30 \%)$ and IOP rise (25-45\%), particularly in younger patients [81, 94]. This should be kept in mind when considering regionally delivered corticosteroids in uveitis patients during pregnancy. Other less common side effects include postinjection infectious endophthalmitis, pseudoendophthalmitis, and rhegmatogenous retinal detachments [95].

In most of these cases, the use of periocular or intravitreal steroid injections has been for the treatment of acute exacerbations, often in combination with the commencement of systemic treatment to prevent the relapse of disease when the sustained release steroid is exhausted. However, due to their limited duration of effect, this modality of treatment tends not to be used as the sole treatment in chronic disease. However, their use during pregnancy would appear ideal, as they have very little (if any) systemic toxicity and only a limited and finite number of repeated administrations would be needed (if required) during the course of the pregnancy, after which systemic treatments could be reconsidered after delivery. Alternatively, newer forms of sustained release corticosteroid therapy such as Ozurdex (Allergan, Irvine, CA) and Retisert (Bausch and Lomb, Rochester, NY) could also be considered during this time, given their longer durations of effect in chronic active posterior or panuveitis, with similar efficacy to systemic treatment $[96,97]$.

A suggested approach in the management of patients with chronic uveitis who become pregnant would therefore be the tapering and cessation of systemic treatments during pregnancy, as the activity of the patient's uveitis would be expected to decrease during this time. Any flare-ups of disease could then be managed locally with either topical, sub-Tenons, or intravitreal sustained release corticosteroid as required until delivery. For those with sight-threatening disease, repeated prophylactic local injections could be considered; however, this would be a more contentious approach, given that disease activity is expected to reduce during pregnancy and common side effects such as raised IOP and cataracts are higher in younger patients [81, 94]. Upon delivery, recommencement of systemic agents (being mindful of the patient's breastfeeding status) and closer review of patients would then be recommended, given that uveitis activity is likely to rebound back to prepregnancy levels. For patients with chronic, sight-threatening disease where the cessation of systemic treatment is deemed particularly risky, an alternative option is the use of either the Ozurdex or Retisert sustained release corticosteroid devices. In those patients planning for multiple children, Retisert may be particularly advantageous, given its much longer duration of effect [98].

\section{Conclusion}

The influence of pregnancy on the course of uveitis is a fascinating phenomenon. The general consensus is that uveitis improves during pregnancy, especially from mid pregnancy onwards, while the postpartum period is associated with uveitis activity relapse. This has key implications on the management of pregnant uveitis patients. Clinicians may consider decreasing uveitis medications during pregnancy to minimise medication associated side effects on the foetus. After delivery, followup should also be intensified in anticipation of postpartum relapse. It would be interesting to see if future studies on the mechanisms behind uveitis amelioration in pregnancy would inspire new therapeutic options for uveitis.

\section{Conflict of Interests}

The authors have no conflict of interests in any aspect of this paper.

\section{Acknowledgment}

CERA receives Operational Infrastructure Support from the Victorian Government. 


\section{References}

[1] J. P. Buyon, J. L. Nelson, and M. D. Lockshin, "The effects of pregnancy on autoimmune diseases," Clinical Immunology and Immunopathology, vol. 78, no. 2, pp. 99-104, 1996.

[2] R. L. Wilder, "Hormones, pregnancy, and autoimmune diseases," Annals of the New York Academy of Sciences, vol. 840, pp. 45-50, 1998.

[3] J. P. Buyon, "The effects of pregnancy on autoimmune diseases," Journal of Leukocyte Biology, vol. 63, no. 3, pp. 281-287, 1998.

[4] A. Doria, L. Iaccarino, S. Arienti et al., "Th2 immune deviation induced by pregnancy: the two faces of autoimmune rheumatic diseases," Reproductive Toxicology, vol. 22, no. 2, pp. 234-241, 2006.

[5] I. J. Elenkov, J. Hoffman, and R. L. Wilder, "Does differential neuroendocrine control of cytokine production govern the expression of autoimmune diseases in pregnancy and the postpartum period?" Molecular Medicine Today, vol. 3, no. 9, pp. 379-383, 1997.

[6] I. J. Elenkov, R. L. Wilder, V. K. Bakalov et al., "IL-12, TNF- $\alpha$, and hormonal changes during late pregnancy and early postpartum: implications for autoimmune disease activity during these times," Journal of Clinical Endocrinology and Metabolism, vol. 86, no. 10, pp. 4933-4938, 2001.

[7] S. O. Keeling and A. E. Oswald, "Pregnancy and rheumatic disease: "by the book" or 'by the doc"' Clinical Rheumatology, vol. 28, no. 1, pp. 1-9, 2009.

[8] L. J. Jara, O. Vera-Lastra, J. M. Miranda, M. Alcala, and J. Alvarez-Nemegyei, "Prolactin in human systemic lupus erythematosus," Lupus, vol. 10, no. 10, pp. 748-756, 2001.

[9] M. Østensen, P. M. Villiger, and F. Förger, "Interaction of pregnancy and autoimmune rheumatic disease," Autoimmunity Reviews, vol. 11, no. 6-7, pp. A437-A446, 2012.

[10] C.-C. Chan, G. F. Reed, Y. Kim, E. Agrón, and R. R. Buggage, "A correlation of pregnancy term, disease activity, serum female hormones, and cytokines in uveitis," British Journal of Ophthalmology, vol. 88, no. 12, pp. 1506-1509, 2004.

[11] R. K. Agarwal, C.-C. Chan, B. Wiggert, and R. R. Caspi, "Pregnancy ameliorates induction and expression of experimental autoimmune uveitis," Journal of Immunology, vol. 162, no. 5, pp. 2648-2654, 1999.

[12] E. F. Foxman, M. Zhang, S. D. Hurst et al., "Inflammatory mediators in uveitis: differential induction of cytokines and chemokines in Th1- versus Th2-mediated ocular inflammation," Journal of Immunology, vol. 168, no. 5, pp. 2483-2492, 2002.

[13] M. Akdis, O. Palomares, W. van de Veen, M. van Splunter, and C. A. Akdis, "TH17 and TH22 cells: a confusion of antimicrobial response with tissue inflammation versus protection," Journal of Allergy and Clinical Immunology, vol. 129, pp. 1438-1449, 2012.

[14] E. Bettelli, M. Oukka, and V. K. Kuchroo, "TH-17 cells in the circle of immunity and autoimmunity," Nature Immunology, vol. 8, no. 4, pp. 345-350, 2007.

[15] J. Furuzawa-Carballeda, M. I. Vargas-Rojas, and A. R. Cabral, "Autoimmune inflammation from the Th17 perspective," Autoimmunity Reviews, vol. 6, no. 3, pp. 169-175, 2007.

[16] M. B. Torchinsky and J. M. Blander, "T helper 17 cells: discovery, function, and physiological trigger," Cellular and Molecular Life Sciences, vol. 67, no. 9, pp. 1407-1421, 2010.

[17] J. Ernerudh, G. Berg, and J. Mjösberg, "Regulatory Thelper cells in pregnancy and their roles in systemic versus local immune tolerance," American Journal of Reproductive Immunology, vol. 66, no. 1, pp. 31-43, 2011.
[18] C. K. Wong, C. Y. Ho, E. K. Li, and C. W. K. Lam, "Elevation of proinflammatory cytokine (IL-18, IL-17, IL-12) and Th2 cytokine (IL-4) concentrations in patients with systemic lupus erythematosus," Lupus, vol. 9, no. 8, pp. 589-593, 2000.

[19] C. Wang, Y. Tian, B. Lei et al., "Decreased IL-27 expression in association with an increased Th17 response in Vogt-KoyanagiHarada disease.," Investigative Ophthalmology \& Visual Science, vol. 53, pp. 4668-4675, 2012.

[20] D. Yen, J. Cheung, H. Scheerens et al., "IL-23 is essential for T cell-mediated colitis and promotes inflammation via IL-17 and IL-6," Journal of Clinical Investigation, vol. 116, no. 5, pp. 13101316, 2006.

[21] W. Wang, S. Shao, Z. Jiao, M. Guo, H. Xu, and S. Wang, "The Th17/Treg imbalance and cytokine environment in peripheral blood of patients with rheumatoid arthritis," Rheumatology International, vol. 32, no. 4, pp. 887-893, 2012.

[22] L. Klotz, S. Burgdorf, I. Dani et al., "The nuclear receptor PPAR $\gamma$ selectively inhibits Th17 differentiation in a T cellintrinsic fashion and suppresses CNS autoimmunity," Journal of Experimental Medicine, vol. 206, no. 10, pp. 2079-2089, 2009.

[23] G. Toldi, J. Rigó Jr., B. Stenczer, B. Vásárhelyi, and A. Molvarec, "Increased prevalence of IL-17-producing peripheral blood lymphocytes in pre-eclampsia," American Journal of Reproductive Immunology, vol. 66, no. 3, pp. 223-229, 2011.

[24] W.-H. Zhu, C.-Z. Lu, Y.-M. Huang, H. Link, and B.-G. Xiao, "A putative mechanism on remission of multiple sclerosis during pregnancy: estrogen-induced indoleamine 2,3-dioxygenase by dendritic cells," Multiple Sclerosis, vol. 13, no. 1, pp. 33-40, 2007.

[25] S. S. Soldan, A. I. A. Retuerto, N. L. Sicotte, and R. R. Voskuhl, "Immune modulation in multiple sclerosis patients treated with the pregnancy hormone estriol," Journal of Immunology, vol. 171, no. 11, pp. 6267-6274, 2003.

[26] Z. Williams, "Inducing tolerance to pregnancy," The New England Journal of Medicine, vol. 367, pp. 1159-1161, 2012.

[27] J. M. Lipton and A. Catania, "Anti-inflammatory actions of the neuroimmunomodulator $\alpha$-MSH," Immunology Today, vol. 18, no. 3, pp. 140-145, 1997.

[28] J. M. Lipton, "Modulation of host defense by the neuropeptide $\alpha$-MSH," Yale Journal of Biology and Medicine, vol. 63, no. 2, pp. 173-182, 1990.

[29] J. Harness, A. Cavanagh, H. Morton, and P. McCombe, "A protective effect of early pregnancy factor on experimental autoimmune encephalomyelitis induced in Lewis rats by inoculation with myelin basic protein," Journal of the Neurological Sciences, vol. 216, no. 1, pp. 33-41, 2003.

[30] T. B. Tomasi Jr., "Structure and function of alpha-fetoprotein," Annual Review of Medicine, vol. 28, pp. 453-465, 1977.

[31] E. Matsuura, Y. Kang, H. Kitakawa et al., "Modulation of T cell function by alpha-fetoprotein: an in vivo study on porcine thyroid peroxidase-induced experimental autoimmune thyroiditis in transgenic mice producing human alpha-fetoprotein," Tumor Biology, vol. 20, no. 3, pp. 162-171, 1999.

[32] S. Vassiliadis, A. Ranella, L. Papadimitriou, A. Makrygiannakis, and I. Athanassakis, "Serum levels of pro- and antiinflammatory cytokines in non-pregnant women, during pregnancy, labour and abortion," Mediators of Inflammation, vol. 7, no. 2, pp. 69-72, 1998.

[33] M. Ostensen, R. Lundgren, G. Husby, and O. P. Rekvig, "Studies on humoral immunity in pregnancy: immunoglobulins, alloantibodies and autoantibodies in healthy pregnant women and in pregnant women with rheumatoid disease," Journal of Clinical and Laboratory Immunology, vol. 11, no. 3, pp. 143-147, 1983. 
[34] C. Taguchi, E. Ikeda, N. Hikita, and M. Mochizuki, "A report of two cases suggesting positive influence of pregnancy on uveitis activity," Nippon Ganka Gakkai zasshi, vol. 103, no. 1, pp. 66-71, 1999.

[35] A. Kubicka-Trzskka, "Endogenous uveitis during pregnancy-a report of 4 cases," Klinika Oczna, vol. 106, no. 3, pp. 328-331, 2004.

[36] K. Yamada, K. Kimoto, J. Ikewaki, K. Nakatsuka, and H. Yatsuka, "A case of recurrent uveitis with remission during pregnancies," Japanese Journal of Clinical Ophthalmology, vol. 57, no. 3, pp. 311-315, 2003.

[37] P. K. Rabiah and A. T. Vitale, "Noninfectious uveitis and pregnancy," American Journal of Ophthalmology, vol. 136, no. 1, pp. 91-98, 2003.

[38] L. I. Kump, R. A. Cervantes-Castañeda, S. N. Androudi, C. S. Foster, and W. G. Christen, "Patterns of exacerbations of chronic non-infectious uveitis in pregnancy and puerperium," Ocular Immunology and Inflammation, vol. 14, no. 2, pp. 99-104, 2006.

[39] N. P. Chiam, A. J. Hall, R. J. Stawell, L. Busija, and L. L. Lim, "The course of uveitis in pregnancy and postpartum," The British Journal of Ophthalmology, vol. 97, no. 10, pp. 1284-1288, 2013.

[40] D. A. Jabs, "Epidemiology of uveitis," Ophthalmic Epidemiology, vol. 15, no. 5, pp. 283-284, 2008.

[41] J. H. Barrett, P. Brennan, M. Fiddler, and A. Silman, "Breastfeeding and postpartum relapse in women with rheumatoid and inflammatory arthritis," Arthritis \& Rheumatism, vol. 43, pp. 1010-1015, 2000.

[42] J. H. Barrett, P. Brennan, M. Fiddler, and A. J. Silman, "Does rheumatoid arthritis remit during pregnancy and relapse postpartum? Results from a nationwide study in the United Kingdom performed prospectively from late pregnancy," Arthritis \& Rheumatism, vol. 42, pp. 1219-1227, 1999.

[43] L. Matera, M. Mori, M. Geuna, S. Buttiglieri, and G. Palestro, "Prolactin in autoimmunity and antitumor defence," Journal of Neuroimmunology, vol. 109, no. 1, pp. 47-55, 2000.

[44] S. E. Walker, D. Miller, D. Hill, and G. R. Komatireddy, "Prolactin, a pituitary hormone that modifies immune responses. Proceedings of the Mini-symposium on Prolactin and SLE, held at the 5th International Conference on Systemic Lupus Erythematosus, Cancun, Mexico.", Lupus, vol. 7, no. 6, pp. 371375, 1998.

[45] K. B. Elbourne, D. Keisler, and R. W. McMurray, "Differential effects of estrogen and prolactin on autoimmune disease in the NZB/NZW F1 mouse model of systemic lupus erythematosus," Lupus, vol. 7, no. 6, pp. 420-427, 1998.

[46] J. L. Nelson and M. Ostenson, "Pregnancy and rheumatoid arthritis," Rheumatic Disease Clinics of North America, vol. 23, no. 1, pp. 195-212, 1997.

[47] M. Østensen and P. M. Villiger, "Immunology of pregnancypregnancy as a remission inducing agent in rheumatoid arthritis," Transplant Immunology, vol. 9, no. 2-4, pp. 155-160, 2002.

[48] S. H. Zrour, R. Boumiza, N. Sakly et al., "The impact of pregnancy on rheumatoid arthritis outcome: the role of maternofetal HLA class II disparity," Joint Bone Spine, vol. 77, no. 1, pp. 36-40, 2010.

[49] E. Musiej-Nowakowska and R. Ploski, "Pregnancy and early onset pauciarticular juvenile chronic arthritis," Annals of the Rheumatic Diseases, vol. 58, no. 8, pp. 475-480, 1999.

[50] M. Ostensen, "Pregnancy in patients with a history of juvenile rheumatoid arthritis," Arthritis and Rheumatism, vol. 34, no. 7, pp. 881-887, 1991.
[51] C. Gordon, "Pregnancy and autoimmune diseases," Best Practice \& Research Clinical Rheumatology, vol. 18, pp. 359-379, 2004.

[52] R. L. Mayock, R. D. Sullivan, R. R. Greening, and R. Jones Jr., "Sarcoidosis and pregnancy," Journal of the American Medical Association, vol. 164, no. 2, pp. 158-163, 1957.

[53] D. A. Snyder and H. H. Tessler, "Vogt-Koyanagi-Harada syndrome," American Journal of Ophthalmology, vol. 90, no. 1, pp. 69-75, 1980.

[54] L. P. Steahly, "Vogt-Koyanagi-Harada syndrome and pregnancy," Annals of Ophthalmology, vol. 22, no. 2, pp. 59-62, 1990.

[55] Z. Friedman, M. Granat, and E. Neumann, "The syndrome of Vogt-Koyanagi-Harada and pregnancy," Metabolic Ophthalmology, vol. 4, no. 3, pp. 147-149, 1980.

[56] M. Nohara, K. Norose, and K. Segawa, "Vogt-Koyanagi-Harada disease during pregnancy," British Journal of Ophthalmology, vol. 79, no. 1, pp. 94-95, 1995.

[57] M. Doi, H. Matsubara, and Y. Uji, "Vogt-Koyanagi-Harada syndrome in a pregnant patient treated with high-dose systemic corticosteroids," Acta Ophthalmologica Scandinavica, vol. 78, no. 1, pp. 93-96, 2000.

[58] N. Miyata, M. Sugita, S. Nakamura et al., "Treatment of VogtKoyanagi- Harada's disease during pregnancy," Japanese Journal of Ophthalmology, vol. 45, no. 2, pp. 177-180, 2001.

[59] D. Wakefield, A. Abu El-Asrar, and P. McCluskey, "Treatment of severe inflammatory eye disease in patients of reproductive age and during pregnancy," Ocular Immunology and Inflammation, vol. 20, pp. 277-287, 2012.

[60] C. Y. Chung, A. K. H. Kwok, and K. L. Chung, "Use of opthalmic medications during pregnancy," Hong Kong Medical Journal, vol. 10, no. 3, pp. 191-195, 2004.

[61] Mycophenolate REMS, "Food and Drug Administration," 2012, https://www.mycophenolaterems.com/HCPOverview.aspx.

[62] M. Barbhaiya and B. L. Bermas, "Evaluation and management of systemic lupus erythematosus and rheumatoid arthritis during pregnancy," Clinical Immunology, vol. 149, pp. 225-235, 2013.

[63] K. K. Temprano, R. Bandlamudi, and T. L. Moore, "Antirheumatic drugs in pregnancy and lactation," Seminars in Arthritis and Rheumatism, vol. 35, no. 2, pp. 112-121, 2005.

[64] A. B. Elliott and E. F. Chakravarty, "Immunosuppressive medications during pregnancy and lactation in women with autoimmune diseases," Women's Health, vol. 6, no. 3, pp. 431442, 2010.

[65] M. Petri, "Immunosuppressive drug use in pregnancy," Autoimmunity, vol. 36, no. 1, pp. 51-56, 2003.

[66] S. R. J. Taylor, H. Isa, L. Joshi, and S. Lightman, "New developments in corticosteroid therapy for uveitis," Ophthalmologica, vol. 224, supplement 1, pp. 46-53, 2010.

[67] P. Ferrante, A. Ramsey, C. Bunce, and S. Lightman, "Clinical trial to compare efficacy and side-effects of injection of posterior sub-Tenon triamcinolone versus orbital floor methylprednisolone in the management of posterior uveitis," Clinical and Experimental Ophthalmology, vol. 32, no. 6, pp. 563-568, 2004.

[68] P. Riordan-Eva and S. Lightman, "Orbital floor steroid injections in the treatment of uveitis," Eye, vol. 8, part 1, pp. 66-69, 1994.

[69] C. J. Helm and G. N. Holland, "The effects of posterior subtenon injection of triamcinolone acetonide in patients with intermediate uveitis," American Journal of Ophthalmology, vol. 120, no. 1, pp. 55-64, 1995.

[70] I. G. M. Duguid, R. L. Ford, S. E. Horgan, H. M. A. Towler, and S. L. Lightman, "Combined orbital floor betamethasone and 
depot methylprednisolone in uveitis," Ocular Immunology and Inflammation, vol. 13, no. 1, pp. 19-24, 2005.

[71] M. L. Dafflon, V. T. Tran, Y. Guex-Crosier, and C. P. Herbort, "Posterior sub-Tenon's steroid injections for the treatment of posterior ocular inflammation: indications, efficacy and side effects," Graefe's Archive for Clinical and Experimental Ophthalmology, vol. 237, no. 4, pp. 289-295, 1999.

[72] V. Tanner, J. J. Kanski, and P. A. Frith, "Posterior sub-Tenon's triamcinolone injections in the treatment of uveitis," Eye, vol. 12, part 4, pp. 679-685, 1998.

[73] M. Roesel, M. Gutfleisch, C. Heinz, B. Heimes, B. Zurek-Imhoff, and A. Heiligenhaus, "Orbital floor triamcinolone acetonide injections for the management of active non-infectious uveitis," Eye, vol. 23, no. 4, pp. 910-914, 2009.

[74] S. Raghava, M. Hammond, and U. B. Kompella, "Periocular routes for retinal drug delivery," Expert Opinion on Drug Delivery, vol. 1, no. 1, pp. 99-114, 2004.

[75] Y. S. Byun and Y.-H. Park, "Complications and safety profile of posterior subtenon injection of triamcinolone acetonide," Journal of Ocular Pharmacology and Therapeutics, vol. 25, no. 2, pp. 159-162, 2009.

[76] M. Roesel, M. Gutfleisch, C. Heinz, B. Heimes, B. ZurekImhoff, and A. Heiligenhaus, "Intravitreal and orbital floor triamcinolone acetonide injections in noninfectious uveitis: a comparative study," Ophthalmic Research, vol. 42, no. 2, pp. 8186, 2009.

[77] R. J. Antcliff, D. J. Spalton, M. R. Stanford, E. M. Graham, T. J. Fytche, and J. Marshall, "Intravitreal triamcinolone for uveitic cystoid macular edema: an optical coherence tomography study," Ophthalmology, vol. 108, no. 4, pp. 765-772, 2001.

[78] S. Young, G. Larkin, M. Branley, and S. Lightman, "Safety and efficacy of intravitreal triamcinolone for cystoid macular oedema in uveitis," Clinical and Experimental Ophthalmology, vol. 29, no. 1, pp. 2-6, 2001.

[79] S. Androudi, E. Letko, M. Meniconi, T. Papadaki, M. Ahmed, and C. S. Foster, "Safety and efficacy of intravitreal triamcinolone acetonide for uveitic macular edema," Ocular Immunology and Inflammation, vol. 13, no. 2-3, pp. 205-212, 2005.

[80] R. I. Angunawela, C. J. Heatley, T. H. Williamson et al., "Intravitreal triamcinalone acetonide for refractory uveitic cystoid macular oedema: longterm management and outcome," Acta Ophthalmologica Scandinavica, vol. 83, no. 5, pp. 595-599, 2005.

[81] H. Kok, C. Lau, N. Maycock, P. McCluskey, and S. Lightman, "Outcome of intravitreal triamcinolone in uveitis," Ophthalmology, vol. 112, no. 11, pp. 1916.e1-1916.e7, 2005.

[82] M. C. Gillies, J. M. Simpson, F. A. Billson et al., "Safety of an intravitreal injection of triamcinolone: results from a randomized clinical trial," Archives of Ophthalmology, vol. 122, no. 3, pp. 336-340, 2004.

[83] S. Tuncer, S. Yilmaz, M. Urgancioglu, and I. Tugal-Tutkun, "Results of Intravitreal Triamcinolone Acetonide (IVTA) injection for the treatment of panuveitis attacks in patients with Behçet disease," Journal of Ocular Pharmacology and Therapeutics, vol. 23, no. 4, pp. 395-401, 2007.

[84] M. Kramer, R. Ehrlich, M. Snir et al., "Intravitreal injections of triamcinolone acetonide for severe vitritis in patients with incomplete Behcet's disease," American Journal of Ophthalmology, vol. 138, no. 4, pp. 666-667, 2004.

[85] M. Karacorlu, B. Mudun, H. Ozdemir, S. A. Karacorlu, and E. Burumcek, "Intravitreal triamcinolone acetonide for the treatment of cystoid macular edema secondary to Behçet disease," American Journal of Ophthalmology, vol. 138, no. 2, pp. 289-291, 2004.

[86] M. Karacorlu, S. Arf Karacorlu, and H. Ozdemir, "Intravitreal triamcinolone acetonide in Vogt-Koyanagi-Harada syndrome," European Journal of Ophthalmology, vol. 16, no. 3, pp. 481-483, 2006.

[87] S. Karacorlu, H. Ozdemir, and M. Karacorlu, "Intravitreal triamcinolone acetonide in serpiginous choroiditis," Japanese Journal of Ophthalmology, vol. 50, no. 3, pp. 290-291, 2006.

[88] H. Ozdemir, M. Karacorlu, and S. Karacorlu, "Intravitreal triamcinolone acetonide in sympathetic ophthalmia," Graefe's Archive for Clinical and Experimental Ophthalmology, vol. 243, no. 7, pp. 734-736, 2005.

[89] R. V. P. Chan, B. D. Seiff, H. A. Lincoff, and D. J. Coleman, "Rapid recovery of sympathetic ophthalmia with treatment augmented by intravitreal steroids," Retina, vol. 26, no. 2, pp. 243-247, 2006.

[90] J. B. Jonas, "Intravitreal triamcinolone acetonide for treatment of sympathetic ophthalmia," American Journal of Ophthalmology, vol. 137, no. 2, pp. 367-368, 2004.

[91] J. B. Jonas and U. H. M. Spandau, "Repeated intravitreal triamcinolone acetonide for chronic sympathetic ophthalmia," Acta Ophthalmologica Scandinavica, vol. 84, no. 3, p. 436, 2006.

[92] M. Roesel, C. Tappeiner, C. Heinz, J. M. Koch, and A. Heiligenhaus, "Comparison between intravitreal and orbital floor triamcinolone acetonide after phacoemulsification in patients with endogenous uveitis," American Journal of Ophthalmology, vol. 147, no. 3, pp. 406-412, 2009.

[93] S. Choudhry and S. Ghosh, "Intravitreal and posterior subtenon triamcinolone acetonide in idiopathic bilateral uveitic macular oedema," Clinical and Experimental Ophthalmology, vol. 35, no. 8, pp. 713-718, 2007.

[94] A. Sallam, R. M. Comer, J. H. Chang et al., "Short-term safety and efficacy of intravitreal triamcinolone acetonide for uveitic macular edema in children," Archives of Ophthalmology, vol. 126, no. 2, pp. 200-205, 2008.

[95] Y. Tao and J. B. Jonas, "Intravitreal triamcinolone," Ophthalmologica, vol. 225, no. 1, pp. 1-20, 2011.

[96] J. H. Kempen, M. M. Altaweel, J. T. Holbrook et al., "Randomized comparison of systemic anti-inflammatory therapy versus fluocinolone acetonide implant for intermediate, posterior, and panuveitis: the multicenter uveitis steroid treatment trial," Ophthalmology, vol. 118, no. 10, pp. 1916-1926, 2011.

[97] C. Lowder, R. Belfort Jr., S. Lightman et al., "Dexamethasone intravitreal implant for noninfectious intermediate or posterior uveitis," Archives of Ophthalmology, vol. 129, no. 5, pp. 545-553, 2011.

[98] L. L. Lim, J. R. Smith, and J. T. Rosenbaum, "Retisert Bausch \& Lomb/control delivery systems," Current Opinion in Investigational Drugs, vol. 6, no. 11, pp. 1159-1167, 2005.

[99] M. Østensen, "Antirheumatic therapy and reproduction. The influence on fertility, pregnancy and breast feeding," Zeitschrift fur Rheumatologie, vol. 65, no. 3, pp. 217-224, 2006.

[100] L. Park-Wyllie, P. Mazzotta, A. Pastuszak et al., "Birth defects after maternal exposure to corticosteroids: prospective cohort study and meta-analysis of epidemiological studies," Teratology, vol. 62, pp. 385-392, 2000.

[101] P. A. Rosandich, J. T. Kelley III, and D. L. Conn, "Perioperative management of patients with rheumatoid arthritis in the era of biologic response modifiers," Current Opinion in Rheumatology, vol. 16, no. 3, pp. 192-198, 2004. 
[102] P. A. Greenberger, Y. K. Odeh, M. C. Frederiksen, and A. J. Atkinson Jr., "Pharmacokinetics of prednisolone transfer to breast milk," Clinical Pharmacology and Therapeutics, vol. 53, no. 3, pp. 324-328, 1993.

[103] L. Ost, G. Wettrell, I. Bjorkhem, and A. Rane, "Prednisolone excretion in human milk," Journal of Pediatrics, vol. 106, no. 6, pp. 1008-1011, 1985.

[104] B. Nørgård, L. Pedersen, K. Fonager, S. N. Rasmussen, and H. T. Sørensen, "Azathioprine, mercaptopurine and birth outcome: a population-based cohort study," Alimentary Pharmacology and Therapeutics, vol. 17, no. 6, pp. 827-834, 2003.

[105] C. Dejaco, C. Mittermaier, W. Reinisch et al., "Azathioprine treatment and male fertility in inflammatory bowel disease," Gastroenterology, vol. 121, no. 5, pp. 1048-1053, 2001.

[106] C. B. Coulam, T. P. Moyer, N. S. Jiang, and H. Zincke, "Breastfeeding after renal transplantation," Transplantation Proceedings, vol. 14, no. 3, pp. 605-609, 1982.

[107] V. T. Armenti, J. S. Radomski, M. J. Moritz et al., "Report from the National Transplantation Pregnancy Registry (NTPR): outcomes of pregnancy after transplantation," Clinical transplants, pp. 97-105, 2001.

[108] P. E. Pergola, A. Kancharla, and D. J. Riley, "Kidney transplantation during the first trimester of pregnancy: immunosuppression with mycophenolate mofetil, tacrolimus, and prednisone," Transplantation, vol. 71, no. 7, pp. 994-997, 2001.

[109] C. Le Ray, A. Coulomb, E. Elefant, R. Frydman, and F. Audibert, "Mycophenolate mofetil in pregnancy after renal transplantation: a case of major fetal malformations," Obstetrics and Gynecology, vol. 103, no. 5, pp. 1091-1094, 2004.

[110] S. Di Paolo, A. Schena, L. F. Morrone et al., "Immunologic evaluation during the first year of life of infants born to cyclosporine-treated female kidney transplant recipients: analysis of lymphocyte subpopulations and immunoglobulin serum levels," Transplantation, vol. 69, no. 10, pp. 2049-2054, 2000.

[111] M. E. Moretti, M. Sgro, D. W. Johnson et al., "Cyclosporine excretion into breast milk," Transplantation, vol. 75, no. 12, pp. 2144-2146, 2003.

[112] J. A. Katz, C. Antoni, G. F. Keenan, D. E. Smith, S. J. Jacobs, and G. R. Lichtenstein, "Outcome of pregnancy in women receiving infliximab for the treatment of Crohn's disease and rheumatoid arthritis," American Journal of Gastroenterology, vol. 99, no. 12, pp. 2385-2392, 2004.

[113] U. Mahadevan, S. Kane, W. J. Sandborn et al., "Intentional infliximab use during pregnancy for induction or maintenance of remission in Crohn's disease," Alimentary Pharmacology and Therapeutics, vol. 21, no. 6, pp. 733-738, 2005.

[114] B. P. Giroir, K. Peppel, M. Silva, and B. Beutler, "The biosynthesis of tumor necrosis factor during pregnancy: studies with a CAT reporter transgene and TNF inhibitors," European Cytokine Network, vol. 3, no. 6, pp. 533-538, 1992.

[115] K. L. Hyrich, D. P. M. Symmons, K. D. Watson, and A. J. Silman, "Pregnancy outcome in women who were exposed to antitumor necrosis factor agents: results from a national population register," Arthritis and Rheumatism, vol. 54, no. 8, pp. 2701-2702, 2006.

[116] D. J. Wallace and M. H. Weisman, "The use of etanercept and other tumor necrosis factor- $\alpha$ blockers in infertility: it's time to get serious," Journal of Rheumatology, vol. 30, no. 9, pp. 18971899, 2003.

[117] G. M. Enns, E. Roeder, R. T. Chan, Z. Ali-Khan Catts, V. A. Cox, and M. Golabi, "Apparent cyclophosphamide, (cytoxan) embryopathy: a distinct phenotype?" American Journal of Medical Genetics, vol. 86, pp. 237-241, 1999.

[118] J. Penso, B. Lippe, R. Ehrlich, and F. G. Smith, “Testicular function in prepubertal and pubertal male patients treated with cyclophosphamide for nephrotic syndrome," Journal of Pediatrics, vol. 84, no. 6, pp. 831-836, 1974.

[119] W. H. B. Wallace, S. M. Shalet, M. Lendon, and P. H. MorrisJones, "Male fertility in long-term survivors of childhood acute lymphoblastic leukaemia," International Journal of Andrology, vol. 14, no. 5, pp. 312-319, 1991.

[120] V. Papadakis, E. Vlachopapadopoulou, K. van Syckle et al., "Gonadal function in young patients successfully treated for Hodgkin disease," Medical and Pediatric Oncology, vol. 32, pp. 366-372, 1999.

[121] P. H. Wiernik and J. H. Duncan, "Cyclophosphamide in human milk," The Lancet, vol. 1, no. 7705, article 912, 1971.

[122] R. Rahimi, S. Nikfar, A. Rezaie, and M. Abdollahi, "Pregnancy outcome in women with inflammatory bowel disease following exposure to 5-aminosalicylic acid drugs: a meta-analysis," Reproductive Toxicology, vol. 25, no. 2, pp. 271-275, 2008.

[123] B. Nørgård, A. E. Czeizel, M. Rockenbauer, J. Olsen, and H. T. Sørensen, "Population-based case control study of the safety of sulfasalazine use during pregnancy," Alimentary Pharmacology and Therapeutics, vol. 15, no. 4, pp. 483-486, 2001.

[124] M. Mogadam, W. O. Dobbins III, B. I. Korelitz, and S. W. Ahmed, "Pregnancy in inflammatory bowel disease: effect of sulfasalazine and corticosteroids on fetal outcome," Gastroenterology, vol. 80, no. 1, pp. 72-76, 1981.

[125] E. Esbjorner, G. Jarnerot, and L. Wranne, "Sulphasalazine and sulphapyridine serum levels in children to mothers treated with sulphasalazine during pregnancy and lactation," Acta Paediatrica Scandinavica, vol. 76, no. 1, pp. 137-142, 1987.

[126] G. Jarnerot and M.-B. Into-Malmberg, "Sulphasalazine treatment during breast feeding," Scandinavian Journal of Gastroenterology, vol. 14, no. 7, pp. 869-871, 1979. 


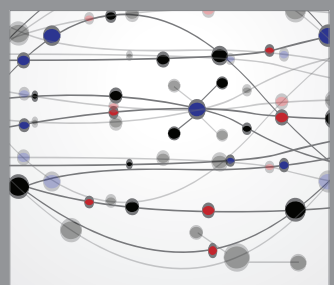

The Scientific World Journal
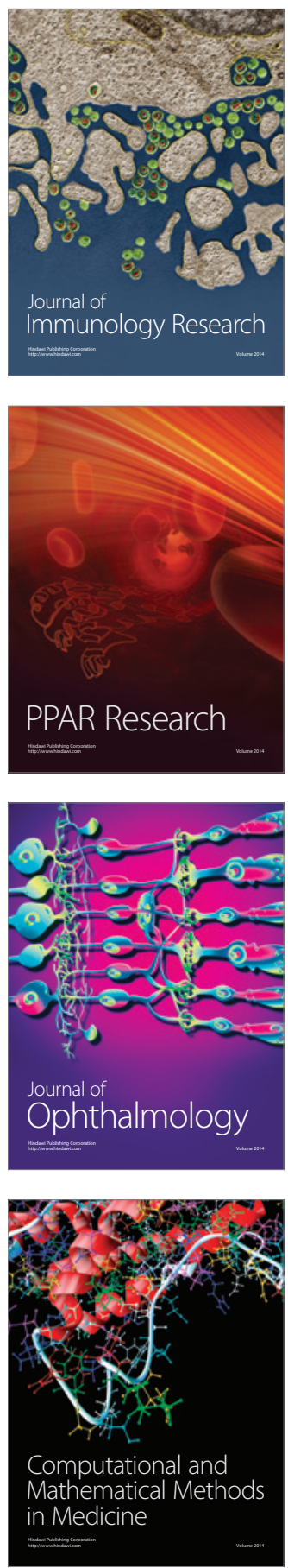

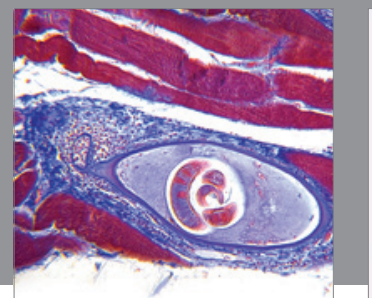

Gastroenterology

Research and Practice
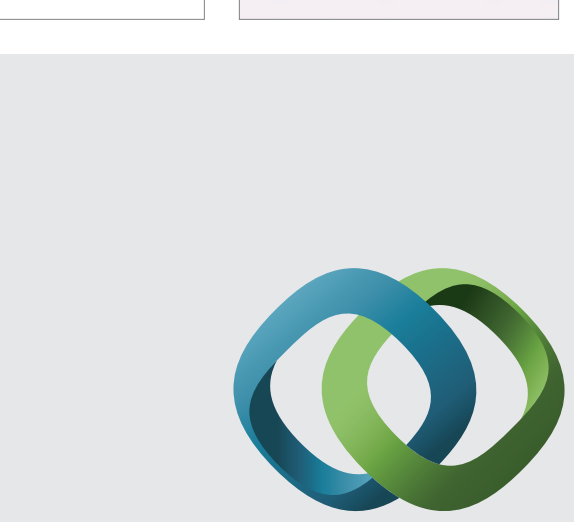

\section{Hindawi}

Submit your manuscripts at

http://www.hindawi.com
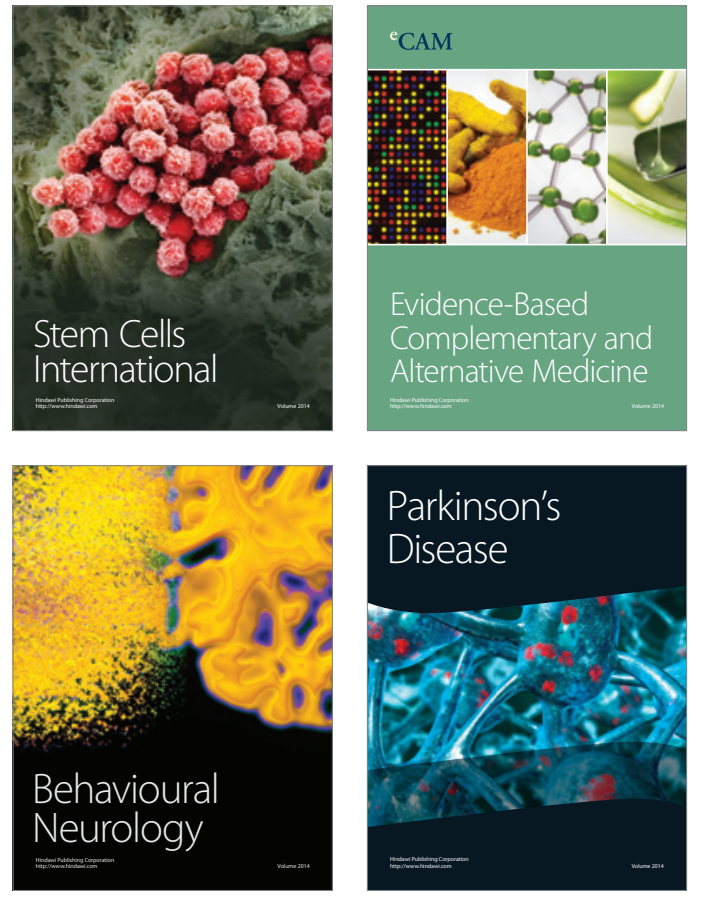
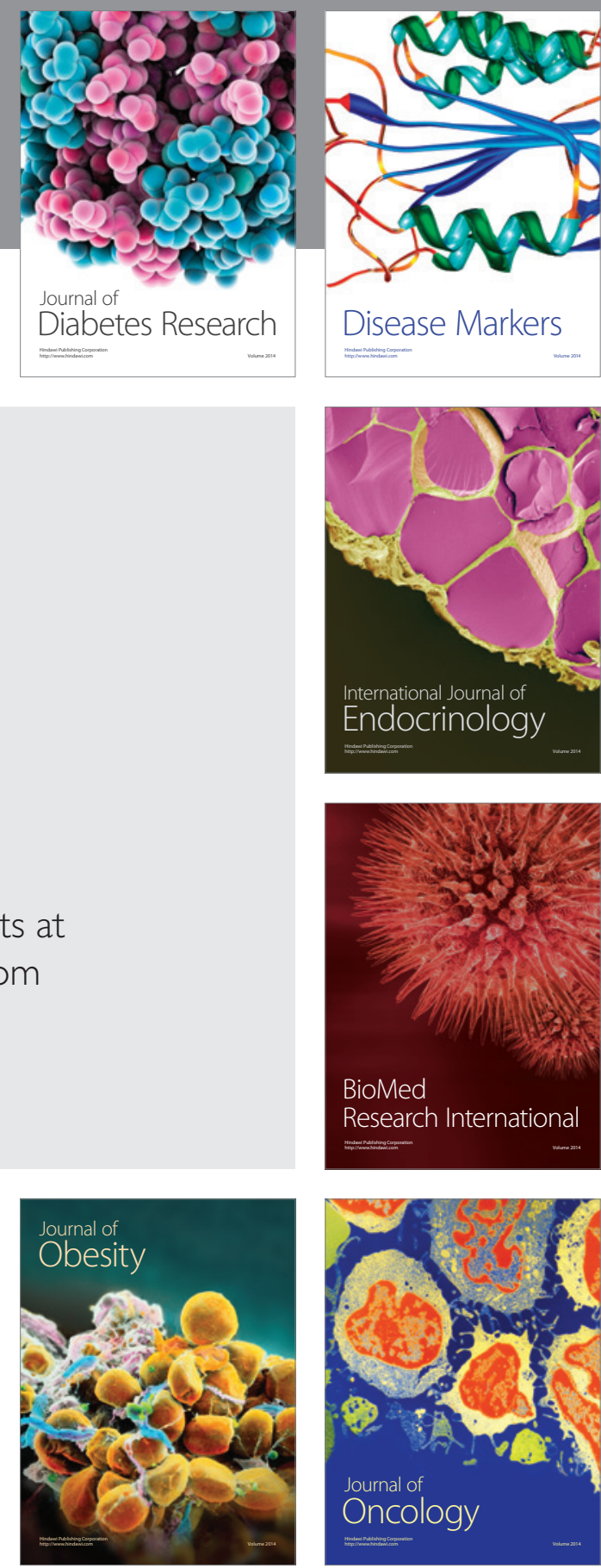

Disease Markers
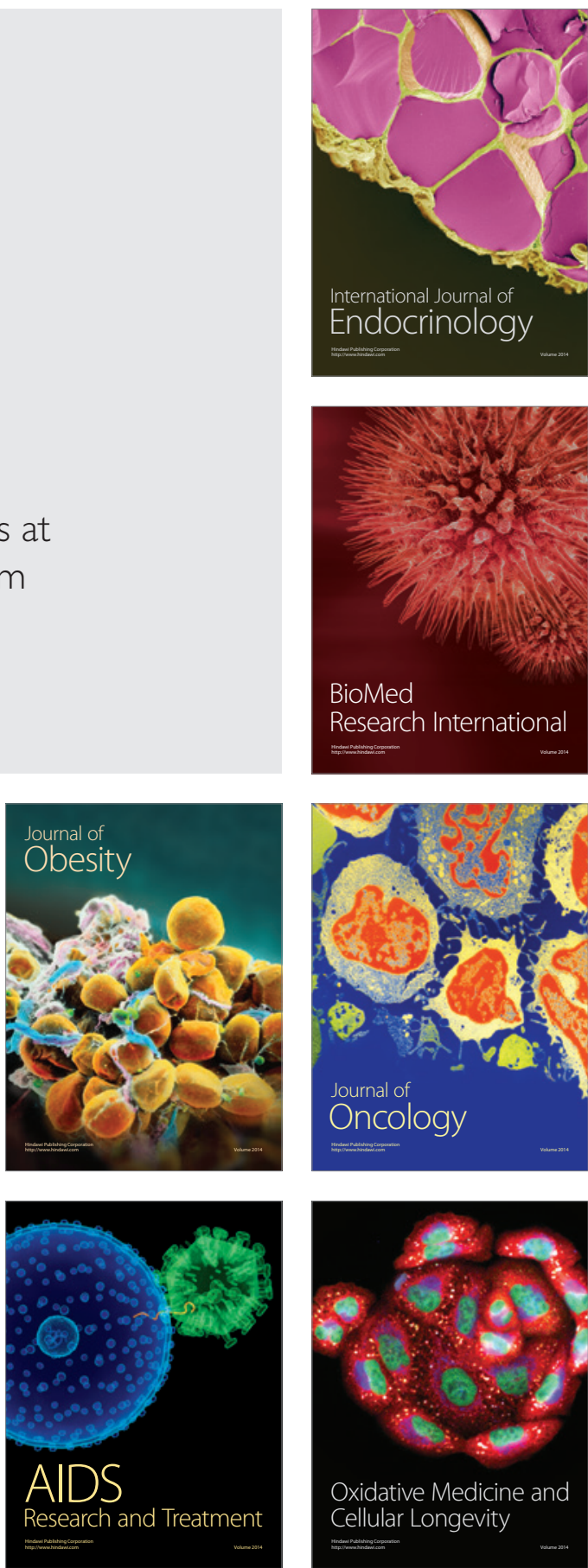